$p<0.01)$, which were also in a concentration-dependent way. 3. The PAI-1 transcriptional activity were significantly suppressed by fenofibrate $(p<0.05)$, but induced by linoleic acid $(p<0.01)$ in HepG2 cells transfected with PAI-pGL3 total length promoter constructs. 4. When co-transfected with PPAR $\alpha$-pSG5, fenofibrate could suppress the level of PAI-1 transcription further more $(p<0.05)$, while increased y linoleic acid $(\mathrm{p}<0.01)$. 5. The PAI-1 transcriptional activity were very inconsistent when transfected with the plasmid containing different length sequences of human PAI-1 gene promoter from -804 to $+17 \mathrm{bp}$.

Conclusions Fenofibrate and linoleic acid could increase the mRNA level of PPAR $\alpha$, and they regulate the synthesis of PAI-1 from transcriptional level, which was concerned with the activated of PPAR $\alpha$ by Fenofibrate and linoleic acid. The sequences that could regulate the expression of PAI-1 gene induced by fenofibrate might exist in the areas from -804 to -636 and -636 to -449 of PAI- 1 promoter and existed in the areas rom -804 to -636 and -449 to -276 induced by linoleic acid. The effects on expression of PAI-1 were very inconsistently, so there might be other mechanisms involved.

\section{e0148 CHANGES IN EXPRESSION OF ERK1/2, ANGIOTENSIN II RECEPTORS IN HIBERNATING MYOCARDIUM}

doi:10.1136/hrt.2010.208967.148

Zhu Hong, Li Dongye, Ding Qian. Affiliated Hospital of Xuzhou Medical College, Xuzhou, Jiangsu, China

Objective To investigate the variation and significance of ERK1/2, Angiotensin II subtype 1 receptor (AT1R) and Angiotensin II subtype 2 receptor $\left(\mathrm{AT}_{2} \mathrm{R}\right)$ in hibernating myocardium.

Methods 6 little domestic Chinese pigs were implanted a constrictor into the right coronary artery through femoral artery to make a immediate $50 \%-75 \%$ stenosis in the target artery. 1 month later after the operation, NTG ${ }^{99} \mathrm{TC}^{\mathrm{m}}$-MIBI SPECT (single photon emission $\mathrm{CT}$ ) was used to detect and locate hibernating myocardium before the animals were killed. Then verify the accuration of SPECT by observing the samples of hibernating myocardium (HM) under electron microscope. Finally assessing the variation of ERK1/ 2, p-ERK1/2 in normal myocardium and HM by western blot, AT1R and $\mathrm{AT}_{2} \mathrm{R}$ were localised by immunohistochemistry and quantified at protein level by western blot respectively.

Results 1 . The spatial distribution of AT1R showed no difference among NM and HM. AT1R were found in myocytes and vascular smooth muscle cells (VSMCs); $\mathrm{AT}_{2} \mathrm{R}$ were found only in myocytes in $\mathrm{NM}$, while in $\mathrm{HM} \mathrm{AT}_{2} \mathrm{R}$ could be found not only in myocytes but also in VSMCs. 2. Compared with NM, the relative amount of AT1R significently reduced in $\mathrm{HM}$ while $\mathrm{AT}_{2} \mathrm{R}$ significently increased in HM. 3. p-ERK1/2 were significantly increased in HM compared with NM.

Conclusion The changes of $\mathrm{AT} 1 \mathrm{R}$ and $\mathrm{AT}_{2} \mathrm{R}$ may help define the pathophysiological role of the angiotensin system in hibernatine myocardium.

\section{e0149 DIAGNOSTIC IMPLICATIONS OF TG/HDL-C AND PTX-3 IN DIAGNOSIS OF ACUTE CORONARY SYNDROME}

doi:10.1136/hrt.2010.208967.149

Zhang Li, Liu Qiming, Zhou Shenghua, Qin Haibin, Zhao Shuiping. The Department of Cardiology, The Second Xiangya Hospital, Central South University, Changsha, China

Objective To investigate the value of serum pentraxin-3 (PTX-3) together with triglyceride (TG)/high-density lipoprotein cholesterol (HDL-C) as a predictor risk factor for future acute coronary syndrome (ACS).

Methods Collected and analysed 171 cases of Department of Cardiology of the Second Xiangya Hospital of Central South
University in July 2008 December 2009 for elective coronary angiography patients, according to the situation on admission and coronary angiography, patients were divided into three groups: normal control subjects, stable angina pectoris and acute coronary syndrome group, all patients admitted to hospital were extracted fasting venous blood for measuring PTX-3 and lipids (TG, HDL-C) level in the next morning, and Statistically analysed, $\mathrm{p}<0.05$ was considered statistically significant.

Results The serum PTX-3 levels and the ratio of TG and HDL-C in patients with acute coronary syndrome $(6.39 \pm 3.01 \mathrm{ng} / \mathrm{ml}$; $2.38 \pm 2.00)$ were significantly increased than those in stable angina pectoris $(3.87 \pm 2.05 \mathrm{ng} / \mathrm{ml} ; 1.70 \pm 1.01)$ and normal control subjects $(2.90 \pm 1.94 \mathrm{ng} / \mathrm{ml}, 0.95 \pm 0.35), \mathrm{p}<0.05$.

Conclusions Increased serum PTX-3 and ratio of TG and HDL-C in patients are closely related with acute coronary syndrome, both increase accuracy of early diagnosis of acute coronary syndrome.

\section{Q0150 HIGH FREQUENCY OF PERI-STRUT LOW INTENSITY AREA ASSESSED BY OPTICAL COHERENCE TOMOGRAPHY AFTER POLYMER-BASED SIROLIMUS-ELUTING STENTS IMPLANTATION IN PORCINE MODEL}

doi:10.1136/hrt.2010.208967.150

Jia Haibo, Hou Jingbo, Wu Jian, Zhang Maomao, Qi Hai, Huang Xingtao, Liu Huiming, Hu Sining, Sun Rong, Zhang Shuo, Yu Bo. Department of Cardiology, Second Affiliated Hospital of Harbin Medical University, Key Laboratories of Education Ministry for Myocardial Ischaemia Mechanism and Treatment, Harbin, China

Objectives To assess the frequency of peri-strut low intensity area (PLIA) in polymeric and nonpolymeric drug-eluting stents by optical coherence tomography (OCT) in porcine model, to analyse the pathological changes in PLIA.

Setting Previous intravascular ultrasound studies showed that black holes were more commonly seen in sirolimus-eluting stent restenosis. Similar phenomenon (peri-strut low intensity area) was also frequently detected by OCT in DES follow-up. However, it is still largely unknown what triggers this uncommon response. Design and interventions: A total of 18 stents (BMS, $n=6$; polymer-free PES [PF-PES], $n=6$ and polymer-based SES [PB-SES], $n=6$ ) were implanted in six minipigs and OCT was performed at 28 days after stenting. Stented arteries were harvested after terminal OCT imaging for pathological analysis. PLIA was defined as a region around stent struts with a homogenous lower intensity appearance than surrounding tissue on OCT images without significant signal attenuation behind the area.

Results At 28 days, PLIA was more frequently observed around the PBSES struts compared with PFPES and BMS struts (75\% vs $33 \%$ vs $12 \%$, respectively, $\mathrm{p}<0.001)$. Both in DES and BMS group, stents with PLIA showed significantly greater neointimal thickness than stents without PLIA $(0.55 \pm 0.23 \mathrm{~mm}$ vs $0.13 \pm 0.08 \mathrm{~mm}, \mathrm{p}<0.001)$. Histological results showed the existence of fibrin deposition and small amount of inflammatory cells at the site of PLIA.

Conclusions PBSES showed a higher incidence of PLIA compared with BMS and PFPES. PLIA may be related to fibrin deposition and vessel chronic inflammatory response to stent.

\section{e0151 EXPRESSION OF IL-17 IN VIRAL INDUCED DILATED CARDIOMYOPATHY MICE}

doi:10.1136/hrt.2010.208967.151

Lin Song, Wu Weifeng. Department of Cardiology, Institute of Cardiovascular Diseases, The First Affiliated Hospital of Guangxi Medical University, Nanning, China

Objective To investigate Interleukin 17 (IL-17) levels in viral dilated cardiomyopathy (DCM) mice, aiming at effect of IL-17 in viral DCM. 